\title{
No-go theorems for functorial localic spectra of noncommutative rings
}

\author{
Benno van den Berg* \\ Mathematisch Instituut \\ Utrecht University \\ B. vandenBerg10uu.nl
}

\author{
Chris Heunen* \\ Department of Computer Science \\ University of Oxford \\ heunen@cs.ox.ac.uk
}

\begin{abstract}
Any functor from the category of $\mathrm{C}^{*}$-algebras to the category of locales that assigns to each commutative $\mathrm{C}^{*}$-algebra its Gelfand spectrum must be trivial on algebras of $n$-by- $n$ matrices for $n \geq 3$. The same obstruction applies to the Zariski, Stone, and Pierce spectra. The possibility of spectra in categories other than that of locales is briefly discussed.
\end{abstract}

A recent article [9] by Reyes shows that any functor CStar $^{\text {op }} \rightarrow$ Top that assigns to each commutative $C^{*}$-algebra its Gelfand spectrum must be trivial on the matrix algebras $\mathbb{M}_{n}(\mathbb{C})$ for $n \geq 3$. More precisely, it shows that any functorial extension of the Gelfand spectrum to noncommutative $\mathrm{C}^{*}$-algebras must yield the empty space on the noncommutative $C^{*}$-algebras $\mathbb{M}_{n}(\mathbb{C})$ for $n \geq 3$. This result shows in a strong way why the traditional notion of topological space is inadequate to host a good notion of spectrum for such noncommutative $\mathrm{C}^{*}$-algebras.

What remains open is whether less orthodox notions of space are also susceptible to Reyes' theorem. In particular, there are notions of space, such as that of a locale or a topos, in which the notion of point plays a subordinate role. Indeed, one of the messages of locale theory and topos theory is that one can have spaces with a rich topological structure, but without any points. Initially, this might seem like an attractive way of circumventing Reyes' result: perhaps there can be a functor $\mathbf{C S t a r}^{\text {op }} \rightarrow \mathbf{L o c}$, extending the Gelfand spectrum for commutative $\mathrm{C}^{*}$-algebras and assigning nontrivial locales to noncommutative $\mathrm{C}^{*}$-algebras such as $\mathbb{M}_{n}(\mathbb{C})$ for $n \geq 3$.

Unfortunately, the main result of this paper says that this is not the case: by sharpening Reyes' argument, one can show that any functor $\mathbf{C S t a r}^{\mathrm{op}} \rightarrow \mathbf{L o c}$ which assigns to a commutative $\mathrm{C}^{*}$-algebra its Gelfand locale must yield the trivial locale (i.e. the locale in which top and bottom element coincide) on the matrix algebras $\mathbb{M}_{n}(\mathbb{C})$ for $n \geq 3$. We obtain similar "no-go theorems" for (ringed) toposes and even for quantales (certain structures which have been put forward as a noncommutative generalization of the notion of locale). Additionally, we prove similar limitative results for Zariski, Stone and Peirce spectra.

This does not mean that it is hopeless to look for a good notion of spectrum of a noncommutative $\mathrm{C}^{*}$ algebra or ring; but what these result do say is that considerable creativity will be required, in particular in finding the right generalisation of the notion of space. We end the paper by mentioning some positive results in this direction.

\section{Locale-theoretic preliminaries}

Locales and topological spaces are closely related apart from a few subtle differences. One of the most important is that in these categories limits are, in general, computed differently. Initially one might hope that for this reason Reyes' result does not apply to locales, but it turns out that it does. The key

${ }^{*}$ Supported by Netherlands Organisation for Scientific Research (NWO).

Bart Jacobs, Peter Selinger, and Bas Spitters (Eds.): 8th International Workshop on Quantum Physics and Logic (QPL 2011) EPTCS 95, 2012, pp. 21-25 doi $10.4204 /$ EPTCS.95.3 (c) B. van den Berg \& C. Heunen

This work is licensed under the Creative Commons Attribution License. 
observation is that, although limits in spaces and locales differ in general, they coincide for those spaces (locales) that arise as spectra.

Proposition 1. Both compact regular and compact completely regular locales are closed under limits in Loc.

Proof. The product of compact (completely) regular locales is again (completely) regular and compact [4, III.1.6, III.1.7, IV.1.5]. The equalizer of $f, g: A \rightarrow B$ is a closed sublocale of $A$ whenever $B$ is (completely) regular [4, III.1.3] and a closed sublocale of a compact (completely) regular locale is again (completely) regular and compact [4, III.1.2, IV.1.5].

Proposition 2. The limit in Loc of a diagram of coherent locales and coherent morphisms is coherent. In addition, the mediating morphisms are coherent.

Proof. A (morphism of) locale(s) is coherent if it lies in the image of the functor Idl: DLat $\rightarrow$ Frm, which is faithful and left adjoint to the forgetful functor [4, II.2.11].

Corollary 3. Stone locales are closed under limits in Loc.

Proof. A locale is Stone when it is both compact regular and coherent. Any continuous morphism between Stone spaces is coherent.

Corollary 4. The functors Spec: cCstar $^{\mathrm{op}} \rightarrow$ Loc and Idl: Bool ${ }^{\mathrm{op}} \rightarrow$ Loc which send commutative $C^{*}$ algebras to their Gelfand spectra and boolean algebras to their Stone spectra preserve all limits.

Proof. The functor Spec is part of a duality between commutative $\mathrm{C}^{*}$-algebras and compact completely regular locales, and therefore certainly preserves all limits as a functor to the category of such locales. But as these locales are closed under limits, it also preserves all limits when regarded as a functor to the category of all locales. The Stone case is completely analogous.

\section{Main results}

A locale is trivial when it is an initial object in Loc, i.e. when it satisfies $0=1$. In categories whose objects contain the matrix rings $\mathbb{M}_{n}(\mathbb{C})$, let us call an object $R$ Kochen-Specker when there is a morphism $\mathbb{M}_{n}(\mathbb{C}) \rightarrow R$ for some $n \geq 3$. Kochen-Specker objects in the category Ring of rings are those rings of the form $\mathbb{M}_{n}(S)$ that allow a ring homomorphism $\mathbb{C} \rightarrow S$ [8, 17.7]. The class of Kochen-Specker objects always includes at least $\mathbb{M}_{n}(\mathbb{C})$ for $n \geq 3$ themselves. For our purposes it can often be widened; for example, by [2], Theorem 7 below still holds when we include in the class of Kochen-Specker algebras the von Neumann algebras without an $\mathbb{M}_{2}(\mathbb{C})$ factor.

Lemma 5. If a functor $F:$ Ring $^{\mathrm{op}} \rightarrow$ Loc is trivial on $\mathbb{M}_{n}(\mathbb{C})$ for all $n \geq 3$, then it is trivial on all Kochen-Specker rings.

Proof. If $f: \mathbb{M}_{n}(\mathbb{C}) \rightarrow R$ is a ring morphism, then $F f: F R \rightarrow F \mathbb{M}_{n}(\mathbb{C})$ is a locale morphism to the trivial locale, and so $F R$ must be trivial.

Theorem 6. Any functor $\mathbf{C s t a r}^{\mathrm{op}} \rightarrow \mathbf{L o c}$ that assigns to each commutative $C^{*}$-algebra its Gelfand spectrum must be trivial on all Kochen-Specker $C^{*}$-algebras. 
Proof. For any $\mathrm{C}^{*}$-algebra $A$, let $\mathscr{C}(A)$ be the diagram of commutative $\mathrm{C}^{*}$-subalgebras under inclusion. Define $G(A)$ to be the limit in $\mathbf{L o c}$ of $\operatorname{Spec}(C)$ with $C \in \mathscr{C}(X)$.

1. $G$ is a functor Cstar $^{\text {op }} \rightarrow$ Loc that assigns to each commutative $\mathrm{C}^{*}$-algebra its Gelfand spectrum.

2. It is the terminal such functor.

3. Since Spec preserves limits, $G(A)$ can equally well be computed by first taking the colimit of $\mathscr{C}(A)$ in cCstar and then its Gelfand spectrum.

4. But for $A=\mathbb{M}_{n}(\mathbb{C})$ with $n \geq 3$, the colimit of $\mathscr{C}(A)$ in cCstar yields the 0 -dimensional $\mathrm{C}^{*}$-algebra; this is the Kochen-Specker theorem [6]. Hence on these $C^{*}$-algebras $G$ yields the trivial locale.

5. If $F:$ Cstar $^{\mathrm{op}} \rightarrow \mathbf{L o c}$ is any other functor that assigns to each $\mathrm{C}^{*}$-algebra its Gelfand spectrum, then finality of $G$ guarantees maps $F A \rightarrow G A$ for all $C^{*}$-algebras $A$. Hence $F A$ is trivial if $A=\mathbb{M}_{n}(\mathbb{C})$ for $n \geq 3$.

Combining the above observations and the previous lemma yields the statement of the theorem.

In a similar vein one proves the following three variations: for Gelfand spectra in the category Neumann of von Neumann algebras and normal *-homomorphisms; for Stone spectra in the category PBoolean of partial boolean algebras and partial homomorphisms (see [6, 1]); and for Stone spectra in the category OML of orthomodular lattices and their homomorphisms. Denote the functor Cstar $\rightarrow$ PBoolean taking projections by Proj.

Theorem 7. Any functor Neumann ${ }^{\text {op }} \rightarrow$ Loc that assigns to each von Neumann algebra its Gelfand spectrum must be trivial on all Kochen-Specker von Neumann algebras.

Theorem 8. Any functor $F:$ PBoolean ${ }^{\mathrm{op}} \rightarrow$ Loc that assigns to each boolean algebra its Stone spectrum must be trivial on $\operatorname{Proj}\left(\mathbb{M}_{n}(\mathbb{C})\right)$ for $n \geq 3$.

Theorem 9. Any functor $\mathbf{O M L}{ }^{\text {op }} \rightarrow$ Loc that assigns to each boolean algebra its Stone spectrum must be trivial on $\operatorname{Proj}\left(\mathbb{M}_{n}(\mathbb{C})\right)$ for $n \geq 3$.

The Pierce spectrum of a commutative ring, i.e. the Stone spectrum of its boolean algebra of idempotents, requires the following slightly adapted proof.

Theorem 10. Any functor Ring ${ }^{\mathrm{op}} \rightarrow$ Loc that assigns to each commutative ring its Pierce spectrum must be trivial on all Kochen-Specker rings.

Proof. Define the functor $G$ as before: it first considers the diagram $\mathscr{C}(R)$ of all commutative subrings of a ring $R$, and then takes the limit of their Pierce spectra. In addition, define three functors $K, L, M$ : Neumann $^{\text {op }} \rightarrow$ Loc: $K$ is simply the composite of $G$ with the inclusion Neumann $\rightarrow$ Ring, while $L$ on a von Neumann algebra $A$ takes the diagram $\mathscr{C}(A)$ of commutative von Neumann subalgebras of $A$, and then takes the limit of their Peirce spectra. Finally, $M$ also takes the diagram $\mathscr{C}(A)$ of commutative von Neumann subalgebras of $A$, but then takes the limit of their Gelfand spectra (which coincides with the Stone spectra on their projections). It is not hard to see that we have natural transformations $K \Rightarrow L$ and $L \Rightarrow M$. But since $M$ results in the trivial locale for $\mathbb{M}_{n}(\mathbb{C})$ for $n \geq 3$ by Theorem 7 , the same must be true for $L, K$ and $G$. And from here the argument proceeds as before.

For the Zariski spectrum we argue slightly differently (and nonconstructively) by reducing the result to the one by Reyes. Let us emphasize that this proof strategy also applies to the previous theorems; but whereas they could also be proven constructively, the Zariski spectrum functor $\mathbf{c R i n g} \rightarrow$ Loc does not preserve limits. 
Theorem 11. Any functor Ring ${ }^{\mathrm{op}} \rightarrow$ Loc that assigns to each commutative ring its Zariski spectrum must be trivial on all Kochen-Specker rings.

Proof. Define the functor $G$ as before, and note that we take a limit of a diagram of coherent locales and coherent morphisms. As such a limit is coherent and coherent locales are spatial (by the prime ideal theorem), its triviality on matrix algebras $\mathbb{M}_{n}(\mathbb{C})$ for $n \geq 3$ follows from the work of Reyes.

\section{Discussion}

Our main results prove an obstruction to direct functorial extensions of various spectra, taking values in locales. There is also no hope for values in categories of which compact completely regular locales are a subcategory that is closed under limits.

- The functor Sh:Loc $\rightarrow$ Topos that takes sheaves preserves limits [5, C.1.4.8], so the obstruction for Loc also holds for Topos.

- The forgetful functor RingedTopos $\rightarrow$ Topos reflects initial objects, so replacing Loc by the category of ringed toposes does not help either. The same holds for ringed spaces, either topological or localic.

- The forgetful functor from the category Scheme of schemes to the category Top of topological spaces reflects initial objects, so there is no use in replacing locales by schemes.

- The category of compact (completely) regular locales is closed under limits in the opposite of the category Quantale of unital quantales and their homomorphisms [7, 4.4]. Using this adapted version of Proposition 1, the proof of Theorem 6 also obstructs functors from taking values in quantales. Similarly, involutive quantales are out of the question.

On the other hand, one could read our main results positively. They guide the search for a 'geometric' spectrum of noncommutative algebras in two ways. We discuss the Gelfand spectrum here, but the underlying ways to overcome the obstacle of our main results hold in general. First, the obstruction can be circumvented by not assigning the Gelfand spectrum to a commutative $\mathrm{C}^{*}$-algebra directly.

- Assigning the quantale of closed linear subspaces to a $\mathrm{C}^{*}$-algebra encodes the Gelfand spectrum of commutative $\mathrm{C}^{*}$-algebras indirectly, and does indeed give a functor [7].

- The Bohrification construction [3, 1], which inspired most of the current work, is not a direct extension of the Gelfand spectrum and hence escapes the hypothesis of our main theorem.

Secondly, there is scope for a functorial spectrum taking values in categories with traditional geometric objects but different morphisms.

- One could consider different morphisms between rings/algebras, and hence take a different view of these objects, to obtain a functorial spectrum resembling a space (see also the discussion in [9. p15]).

- For example, there is an interesting functor $F$ from Cstar to the category of quantum frames, that for commutative $\mathrm{C}^{*}$-algebras comes down to the Gelfand spectrum [10]. This does not contradict the above results, because there is no forgetful functor from quantum frames to either quantales or locales: indeed, $F\left(\mathbb{M}_{3}(\mathbb{C})\right)$ consists of closed right ideals of $\mathbb{M}_{3}(\mathbb{C})$, and therefore is not trivial. 


\section{References}

[1] B. van den Berg \& C. Heunen (2011): Noncommutativity as a colimit, doi:10.1007/s10485-011-9246-3. To appear in Applied Categorical Structures. Available as arXiv:1003.3618.

[2] A. Döring (2005): Kochen-Specker theorem for Von Neumann algebras. International Journal of Theoretical Physics 44(2), pp. 139-160, doi:10.1007/s10773-005-1490-6.

[3] C. Heunen, N.P. Landsman \& B. Spitters (2011): Bohrification. In: Deep Beauty-Understanding the Quantum World through Mathematical Innovation, Cambridge University Press, doi:10.1017/ CB09780511976971.008.

[4] P.T. Johnstone (1982): Stone spaces. Cambridge studies in advanced mathematics 3, Cambridge University Press.

[5] P.T. Johnstone (2002): Sketches of an elephant: A topos theory compendium. Oxford University Press.

[6] S. Kochen \& E. Specker (1967): The problem of hidden variables in quantum mechanics. Journal of Mathematics and Mechanics 17, pp. 59-87, doi:10.1512/iumj.1968.17.17004.

[7] D. Kruml, J.W. Pelletier, P. Resende \& J. Rosicky (2003): On quantales and spectra of $C^{*}$-algebras. Applied Categorical Structures 11(6), pp. 543-560, doi:10.1023/A : 1026106305210.

[8] T.Y. Lam (1999): Lectures on Modules and Rings. Graduate texts in mathematics 189, Springer.

[9] M. L. Reyes (2011): Obstructing extensions of the functor Spec to noncommutative rings. To appear in the Israel Journal of Mathematics. Available as arXiv:1101.2239.

[10] J. Rosicky (1989): Multiplicative lattices and $C^{*}$-algebras. Cahiers de topologie et géométrie différentielle catégoriques 30(2), pp. 95-110. Available at http://www .numdam.org/item?id=CTGDC_1989__30_2_ 95_0. 